\title{
Detection of myocardial ischaemia using surface microdialysis on the beating heart
}

\author{
Pernilla Abrahamsson ${ }^{1}$, Anna-Maja Åberg ${ }^{1}$, Göran Johansson ${ }^{1}$, Ola Winsö ${ }^{1}$, Anders Waldenström² \\ and Michael Haney ${ }^{1}$ \\ ${ }^{1}$ Department of Surgical and Perioperative Sciences, Anaesthesiology and Intensive Care Medicine, University Hospital of Umeå, Umeå, Sweden, and ${ }^{2}$ Department of \\ Public Health and Clinical Medicine, Cardiology, University Hospital of Umeå, Umeå, Sweden
}

\section{Summary}

\section{Correspondence}

Pernilla Abrahamsson, Department of Surgical and Perioperative Sciences, Anesthesiology and Intensive Care Medicine, University Hospital of Umeå, SE-901 85 Umeå, Sweden

E-mail: pernilla.abrahamsson@anestesi.umu.se

\section{Accepted for publication}

Received 15 June 2010;

accepted 20 October 2010

\section{Key words}

epicardium; heart; ischaemia; metabolism; microdialysis; myocardium
Microdialysis (MD) can be used to study metabolism of the beating heart. We investigated whether microdialysis results obtained from epicardial (surface) sampling reflect acute changes in the same way as myocardial sampling from within the substance of the ventricular wall. In anaesthetized open-thorax pigs a coronary snare was placed. One microdialysis probe was placed with the sampling membrane intramyocardially (myocardial), and a second probe was placed with the sampling membrane epicardially (surface), both in the area which was made ischaemic. Ten minutes collection intervals were used for microdialysis samples. Samples from 19 pigs were analysed for lactate, glucose, pyruvate and glycerol during equilibration, baseline, ischaemia and reperfusion periods. For both probes (surface and myocardial), a total of 475 paired simultaneous samples were analysed. Results from analyses showed no differences in relative changes for glucose, lactate and glycerol during baseline, ischaemia and reperfusion. Surface microdialysis sampling is a new application of the microdialysis technique that shows promise and should be further studied.

\section{Introduction}

Microdialysis is a research sampling tool which can be used to detect ischaemia in a solid organ. Microdialysis facilitates serial biochemical sampling through a probe which lies within the substance of an organ. Serial sample collection is based on passive diffusion through a semi-permeable membrane placed at the end of the sampling probe (Ungerstedt, 1991; Chaurasia, 1999; Stahl et al., 2002; Kavianipour et al., 2003; Waldenström et al., 2010).

Microdialysis sampling can be performed in organs which move, and this technique has been used to study metabolic aspects of the beating heart (Kavianipour et al., 2003; Waldenström et al., 2010). How and exactly where the membrane is situated is not easy to determine since it lies in the myocardium and the membrane can no longer be visualized. When a microdialysis probe is placed in the substance of a beating heart, there is always concern for disturbance by heart motion for probe position and stability over time. There is risk for damage to heart tissue as well as damage to the probe when sharply inserting the microdialysis probe (Mantovani et al., (C) 2010 The Authors

Clinical Physiology and Functional Imaging (C) 2010 Scandinavian Society of Clinical Physiology and Nuclear Medicine 31, 3, 175-181
2002; Kavianipour et al., 2003). Despite these concerns, Langemann et al. (1996) have successfully placed the microdialysis membrane in the left ventricular wall for measurements of glucose and lactate concentrations. Microdialysis has shown promise for clinical myocardial metabolic sampling (Kennergren et al., 2003; Pöling et al., 2007a,b, 2008) but is more frequently used in experimental settings.

There is an obligatory time period to observe after probe placement before there can be adequate sampling and this is related to sharp insertion and presumed minimal haematoma presence surrounding the membrane. A $30-150 \mathrm{~min}$ period is typically observed to allow samples to establish reliable equilibration with local myocardial cell conditions (Bolinder et al., 1989; Mantovani et al., 2002, 2006; Flodgren et al., 2005; Pöling et al., 2007a,b, 2008).

We hypothesized that sampling with a microdialysis membrane placed on the solid organ surface can be used to identify metabolic substance concentration changes induced by ischaemia. We further hypothesized that there is a more rapid equilibration for surface sampling compared to probe membranes in the substance of the heart muscle. We aimed to test 
this by comparing results from surface microdialysis sampling to traditional sampling from within the left ventricular wall in a large animal in vivo model of controlled myocardial ischaemia.

\section{Methods}

The study was approved by the Animal Experimental Ethics Committee at Umeå University Sweden, and was conducted in accordance with the (Guide for the Care and Use of Laboratory Animals, National Research Council, Washington, USA, 1996) NIH Institutional animal care and use committee guidebook.

\section{Preparation}

Pigs were premedicated with an intramuscular injection of xylazine $20 \mathrm{mg} \mathrm{kg}^{-1}$ (Rompum vet, Bayer AB, Lyngby, Denmark), atropine sulphate $0.05 \mathrm{mg} \mathrm{kg}^{-1}$ (Atropin, NM, Pharma, Stockholm, Sweden) and ketamine $10 \mathrm{mg} \mathrm{kg}^{-1}$ (Ketalar ${ }^{\circledR}$, Pfizer, Morris Plains, NJ, USA). An intravenous bolus dose of pentobarbital $10 \mathrm{mg} \mathrm{kg}^{-1}$ (Pentobarbitalnatrium, Apoteksbolaget, Stockholm, Sweden) induced anaesthesia, and for maintenance of anaesthesia; midazolam $0.3 \mathrm{mg} \mathrm{kg}^{-1} \mathrm{~h}^{-1}$ (Dormicum, Roche, Basel, Switzerland), pentobarbital $5 \mathrm{mg} \mathrm{kg}^{-1} \mathrm{~h}^{-1}$ and fentanyl $20 \mu \mathrm{g} \mathrm{kg}^{-1} \mathrm{~h}^{-1}$ (Fentanyl, Braun, Melsungen, Germany), were infused. The animals were tracheotomized (7.0 OD endotracheal tube, Rusch, Kernen, Germany) and mechanically ventilated to normoxia and normocapnia (Evita 4, Dräger, Kiel, Germany) as judged by intermittent arterial blood gas analyses (ABL 5 autoanalyzer, Radiometer, Denmark). Ringer's acetate (Pharmacia, Upjohn, Sweden) was infused at $15 \mathrm{ml} \mathrm{kg}{ }^{-1} \mathrm{~h}^{-1}$. A catheter was placed in the external small neck artery and a central venous catheter was inserted in the external jugular vein. Circulatory variables were continuously registered including mean arterial pressure (MAP), heart rate (HR), and central venous pressure (CVP) using a digital signal acquisition and analysis software (Acknowledge; Biopac System Inc., Santa Barbara, CA, USA).

Through a median sternotomy, a diagonal branch of the left anterior descending (LAD) artery was identified and a suture snare was placed proximally. One microdialysis probe (CMA 20; CMA Microdialysis, Solna, Sweden) was placed with the membrane in the substance of the myocardial tissue supplied by the snared LAD branch, using a plastic introducer, this probe labelled myocardial. A second microdialysis probe was placed and secured on the epicardial surface above the first myocardial probe (labelled surface).

\section{Experimental protocol}

Microdialysis probes were perfused with a modified KrebsRinger phosphate buffer, Fresenius Kabi, Halden, Norway with a flow rate of $2.0 \mu \mathrm{lmin}^{-1}$. Samples were collected at $10 \mathrm{~min}$ intervals throughout the study protocol. After an equilibrium period of 60 min during which MD sample were collected and analysed animals were randomized in two groups according to a protocol for another study. One group, $(\mathrm{n}=10)$ labelled protocol 1, had a 150-min period without any intervention (baseline) before $50 \mathrm{~min}$ of ischaemia induced by tightening the snare, control group. The second group, $(n=9)$ labelled protocol 2, had four 10 min periods of snare occlusion, each followed by 20 min of reperfusion (short ischaemic period), before the $50 \mathrm{~min}$ ischaemic period (long ischaemic period).

\section{Analyses}

Fifty microdialysis samples/vials (20 $\mu \mathrm{l}$ in each) were collected pair-wise for each animal, 25 from each probe. A total of 475 paired samples were compared from the four periods, 95 samples for equilibration, 38 samples for baseline, 135 samples for short ischaemia and 95 samples for long ischaemia period. Glucose, lactate, pyruvate and glycerol were analysed in a CMA 600 analyzer (CMA). All samples were collected in glass vials and sealed with a crimp caps (Chromacol, CT, USA) directly after sampling. The samples were stored in $6^{\circ} \mathrm{C}$ no longer than 1 day. All samples were centrifuged (Mini Galaxy; VWR, West Chester, PA, USA) for $30 \mathrm{~s}$ (1177 g) before analyses (Abrahamsson et al., 2008).

\section{Statistical analysis}

Measured values are presented as mean and standard error of the mean $(\mathrm{SEM})$. A paired t-test was performed $(\mathrm{P}<0.05)$ for the absolute concentrations. A paired comparison to measure differences in relative changes between probes was calculated with a 95\% confidential interval. SPSS (SPSS Inc., Chicago, IL, USA) was used to all statistical analysis.

\section{Results}

A total of 20 female juvenile pigs $(28-45 \mathrm{~kg})$ were studied. One pig was excluded due to ventricular fibrillation. The sampling protocols and interventions are illustrated in Figs 1a,b-4a,b and presented as relative changes from the two first baseline points (B). The first sample (0-10 min) was excluded because it was a mixed (perfusion fluid and fluid from the tissue) sample. Samples numbered 2-6 are grouped into an equilibration period (E). Baseline values (B) were calculated as the group mean of the two first samples (samples 7 and 8) separately for both protocols. Samples 9-21 in protocol 1 depict a prolonged baseline period without any intervention. Data reflecting the short ischaemic periods (I) were calculated as a group mean of the four peak values in protocol 2 (Samples 9-21). Data reflecting the long ischaemic periods (I) were calculated as is a mean of the last sample during the final ischaemic period (sample 26) separately for both protocols.

\section{Lactate}

Absolute concentrations at baseline were significantly higher in samples obtained from the surface probe compared with

Clinical Physiology and Functional Imaging (C) 2010 Scandinavian Society of Clinical Physiology and Nuclear Medicine 31, 3, 175-181 

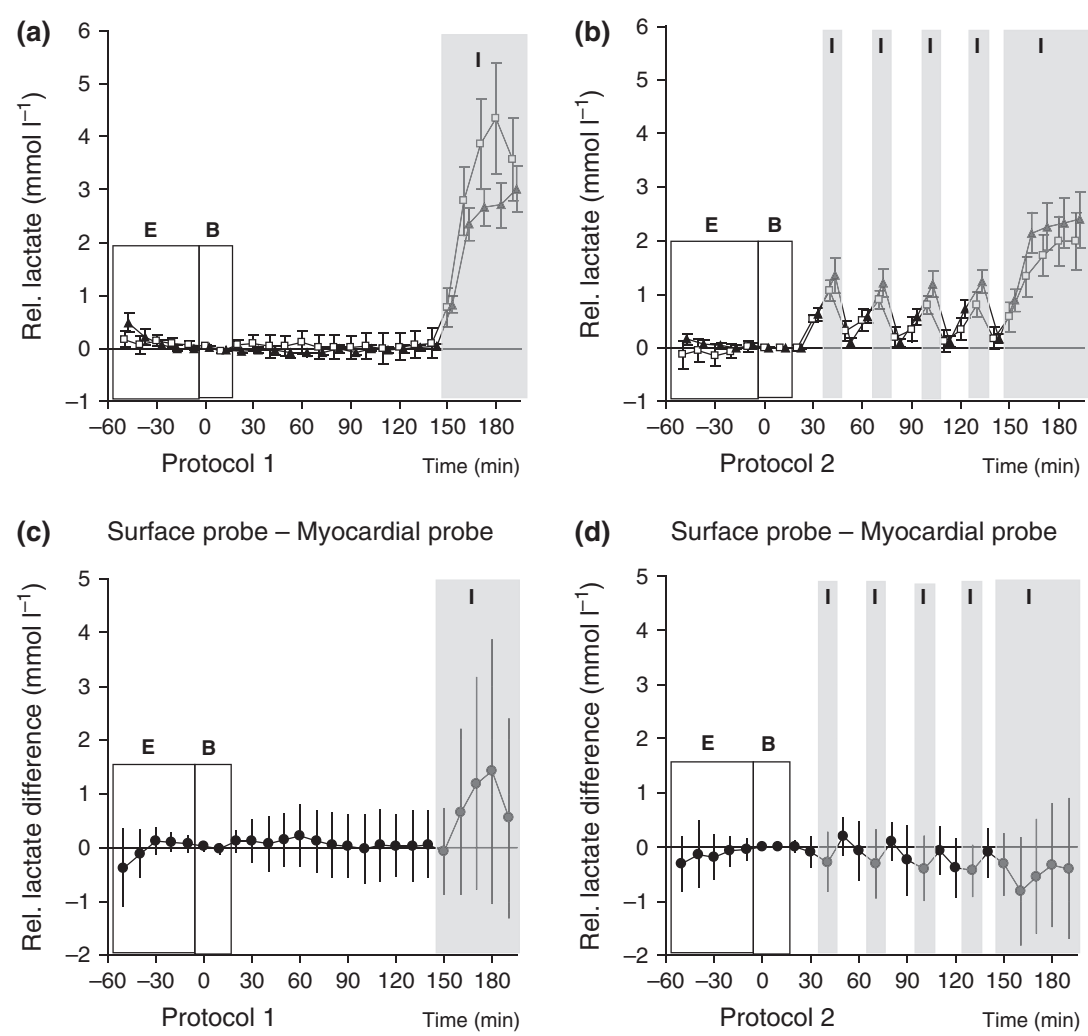

Figure 1 Lactate. Panels (a) and (b) depicts relative changes in lactate concentrations from baseline (time 0) based on data obtained from the surface probe (open squares) and the myocardial probe (filled triangles). Panels (c) and (d) depicts paired comparisons of relative changes in lactate concentrations between the surface probe and the myocardial probe at corresponding time points (filled circles). Data are presented as mean \pm SEM (panels a and b) and as mean $\pm 95 \%$ confidence intervals (panels $\mathrm{c}$ and $\mathrm{d}$ ). $\mathrm{E}=$ Equilibration time, $\mathrm{B}=$ Baseline, $\mathrm{I}=$ Ischaemia. Protocol $1, \mathrm{n}=10$ and protocol $2, \mathrm{n}=9$.

samples from the myocardial probe in both protocol 1 and 2 (Table 1). Both probes showed increased and decreased relative changes in concentrations during ischaemia and reperfusion, respectively (Fig. 1a,b). A comparison between relative changes in lactate concentrations obtained from both probes reviled a similar pattern throughout the study protocol (Fig. 1c,d).

\section{Glucose}

Table 1 showed that the glucose concentration was not significantly higher in the surface probe compared with the glucose concentration in the myocardial probe for both protocol 1 and 2. Relative changes in glucose concentrations obtained from both probes showed a similar pattern, with no significantly difference between the surface and myocardial probe during baseline, short ischaemia and long ischaemia. Note that the surface probe did not show the same pattern (though inverted) as the myocardial probe with decreased and then increased glucose concentrations during short ischaemia periods and reperfusion (Fig. 2a-d).

\section{Pyruvate}

Absolute values for pyruvate concentrations were significantly higher in samples obtained from the surface probe during (C) 2010 The Authors

Clinical Physiology and Functional Imaging (C 2010 Scandinavian Society of Clinical Physiology and Nuclear Medicine 31, 3, 175-181 baseline and long ischaemic period for both protocol 1 and 2 (Table 1). There was a tendency towards higher concentrations in samples obtained from the myocardial probe during the long baseline period in protocol 1 , but not during any of the other periods, i.e. ischaemia and reperfusion (Fig. 3a-d).

\section{Glycerol}

Absolute glycerol concentrations in samples obtained from the control probe were significantly higher than concentrations in samples from the surface probe during the long ischaemic period in protocol 1 (Table 1). There were no other significant differences in absolute concentrations between samples obtained from both probes. A comparison between relative changes in glycerol concentrations obtained from both probes revealed a similar pattern throughout the study (Fig. 4a-d).

\section{Discussion}

The main findings were that results from the surface probe samples followed closely both in time and magnitude, the myocardial metabolic events related to acute ischaemia as demonstrated by samples from a probe placed in the ventricular wall. We have also shown that detection of rapid changes was possible with surface sampling, especially for lactate, even with 
Table 1 Absolute concentrations in microdialysate samples from a probe on the surface $(S)$ of the heart and from a probe in the myocardium (M) during baseline (time 0), the four short ischaemic periods and at the end of the long ischaemic period.

\begin{tabular}{lccc}
\hline Substance & Baseline & $\begin{array}{c}\text { Short } \\
\text { ischemia }\end{array}$ & $\begin{array}{c}\text { Long } \\
\text { ischemia }\end{array}$ \\
\hline Protocol 1 & & & \\
Lactate - S $(\mathrm{mM})$ & $1.46 \pm 0.59 *$ & & $5.03 \pm 1.44$ \\
Lactate - M $(\mathrm{mM})$ & $1.01 \pm 0.36$ & & $4.03 \pm 1.11$ \\
Glucose - S $(\mathrm{mM})$ & $2.00 \pm 0.67$ & & $0.42 \pm 0.32$ \\
Glucose - M (mM) & $1.76 \pm 0.37$ & & $0.29 \pm 0.23$ \\
Pyruvate - S $(\mu \mathrm{M})$ & $71.3 \pm 18.7 *$ & & $92.9 \pm 40.2 *$ \\
Pyruvate - M $(\mu \mathrm{M})$ & $26.0 \pm 11.9$ & & $33.7 \pm 26.2$ \\
Glycerol - S $(\mu \mathrm{M})$ & $54.0 \pm 37.5$ & & $127.8 \pm 44.4$ \\
Glycerol - M $(\mu \mathrm{M})$ & $57.4 \pm 36.8$ & & $201.3 \pm 94.9 *$ \\
Protocol 2 & & & \\
Lactate - S $(\mathrm{mM})$ & $1.42 \pm 0.34 *$ & $2.32 \pm 0.56$ & $3.44 \pm 1.22$ \\
Lactate - M $(\mathrm{mM})$ & $0.83 \pm 0.23$ & $2.08 \pm 0.67$ & $3.17 \pm 1.30$ \\
Glucose - S $(\mathrm{mM})$ & $2.60 \pm 0.90$ & $1.77 \pm 0.41$ & $0.75 \pm 0.73$ \\
Glucose - M $(\mathrm{mM})$ & $2.06 \pm 0.48$ & $1.27 \pm 0.41$ & $0.42 \pm 0.30$ \\
Pyruvate - S $(\mu \mathrm{M})$ & $65.8 \pm 28.0 *$ & $102.0 \pm 46.8$ & $111.6 \pm 35.3 *$ \\
Pyruvate - M $(\mu \mathrm{M})$ & $31.2 \pm 10.9$ & $60.4 \pm 18.1$ & $51.9 \pm 24.6$ \\
Glycerol - S $(\mu \mathrm{M})$ & $46.3 \pm 35.3$ & $38.3 \pm 24.4$ & $46.0 \pm 32.7$ \\
Glycerol - M $(\mu \mathrm{M})$ & $39.7 \pm 38.3$ & $43.0 \pm 33.7$ & $64.6 \pm 45.8$ \\
\hline
\end{tabular}

The baseline values were calculated as the group mean of the first two samples, short ischaemia is a grouped mean over the four peak values and the long ischaemia is a mean over the last sample during the ischaemic period. ischaemic periods as short as $10 \mathrm{~min}$. The MD technique is not based on absolute concentrations but rather on relative concentrations. Relative changes in glucose, lactate, pyruvate and glycerol showed no significant differences between surface and myocardial probe during baseline, short ischaemia and long ischaemia.

The evaporation process might affect a surface probe exposed to air, as was the case in our model. While there were higher concentrations for surface measurements of lactate, pyruvate and glucose, this was not the case for glycerol. In theory, there is a fluid viscous that surrounds the membrane portion which is in contact with air where evaporation can occur. The glycerol finding does not support the interpretation of evaporation as an explanation of the higher concentration for the surface probe results. The effect of evaporative forces and the relation of probe membrane, tissue, and local fluid need to be further evaluated. This study was not designed to assess evaporative sites and forces specifically.

There was some disagreement between probes during the equilibration period which narrowed over time particularly for glucose, pyruvate and glycerol, with glycerol values reaching strong agreement already at the end of the equilibration period. Unexpectedly, perhaps the best agreement for lactate appeared to occur during the very first measurement period.

One desirable performance aspect for a clinically used microdialysis assessment system is a short equilibration time. Previous studies have shown equilibration times varying between 30 and 150 min (Bolinder et al., 1989; Mantovani et al., 2002; Flodgren

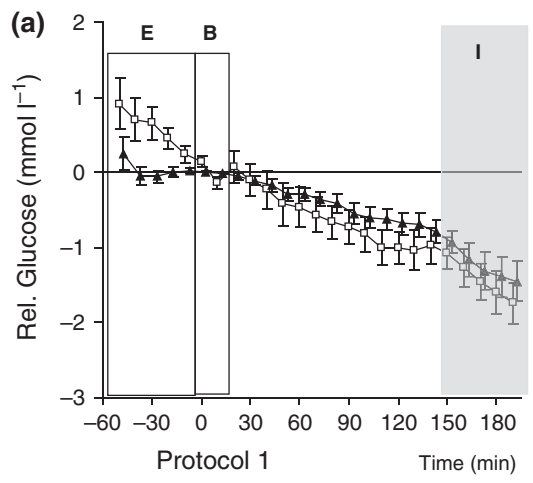

(c) Surface probe - Myocardial probe

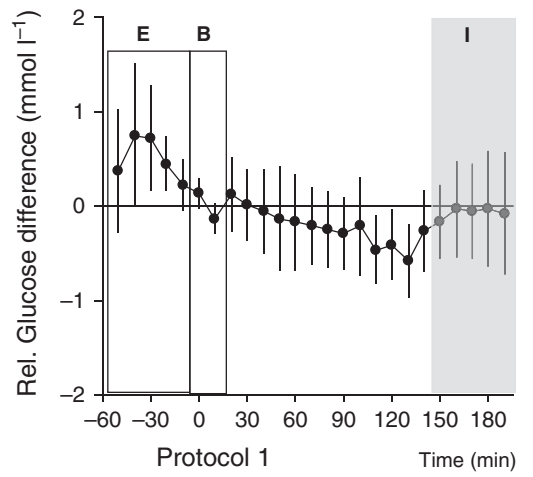

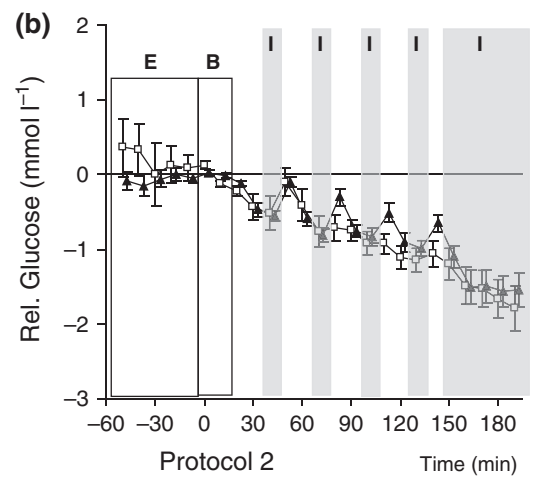

(d) Surface probe - Myocardial probe

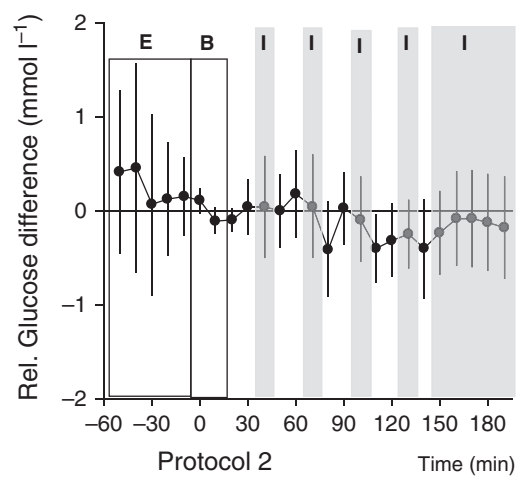

Figure 2 Glucose. Panels (a) and (b) depicts relative changes in glucose concentrations from baseline (time 0) based on data obtained from the surface probe (open squares) and the myocardial probe (filled triangles). Panels (c) and (d) depicts paired comparisons of relative changes in glucose concentrations between the surface probe and the myocardial probe at corresponding time points (filled circles). Data are presented as mean \pm SEM (panels a and b) and as mean $\pm 95 \%$ confidence intervals (panels $\mathrm{c}$ and $\mathrm{d}) . \mathrm{E}=$ Equilibration time, $\mathrm{B}=$ Baseline, $\mathrm{I}=$ Ischaemia. Protocol $1, \mathrm{n}=10$ and protocol $2, \mathrm{n}=9$.

(C) 2010 The Authors

Clinical Physiology and Functional Imaging (C) 2010 Scandinavian Society of Clinical Physiology and Nuclear Medicine 31, 3, 175-181 

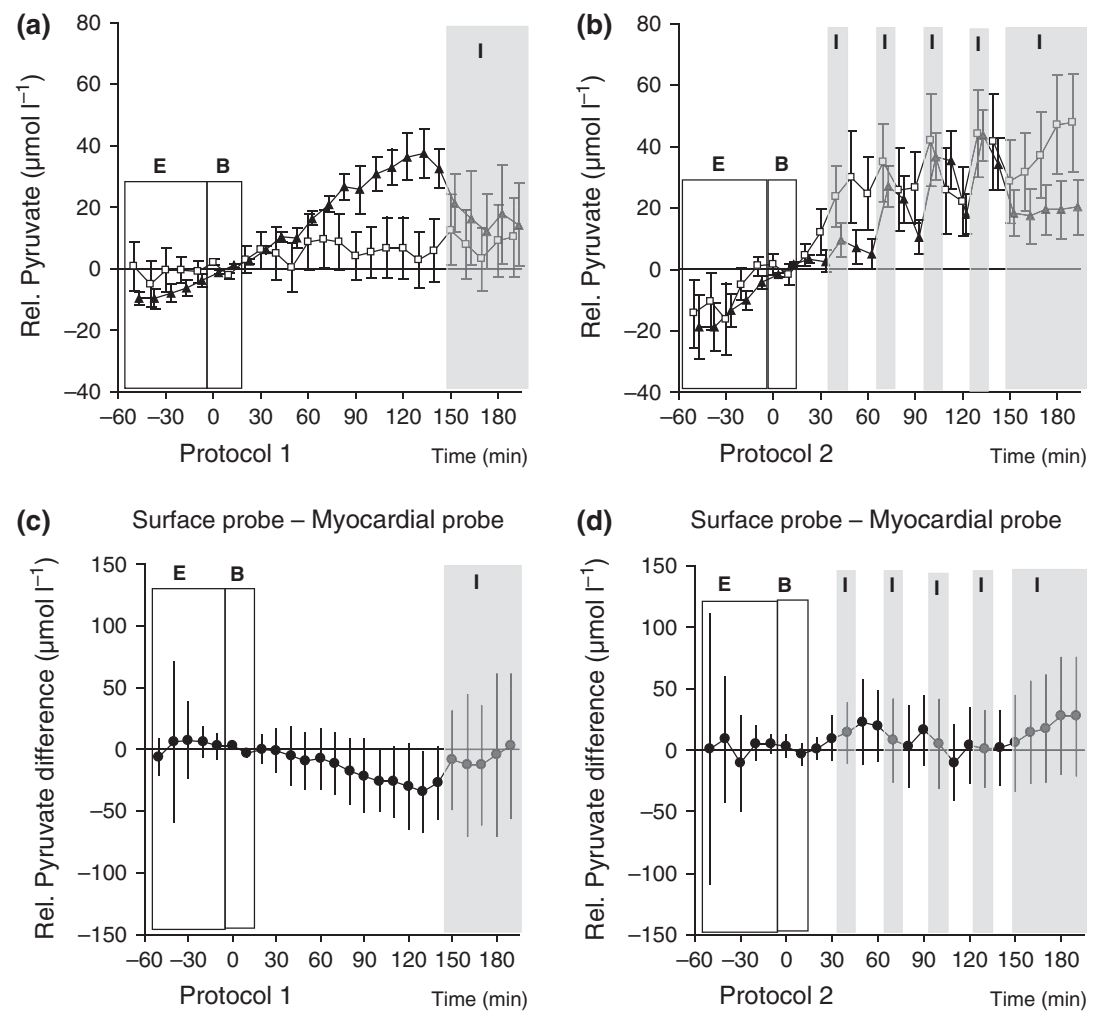

Figure 3 Pyruvate. Panels (a) and (b) depicts relative changes in pyruvate concentrations from baseline (time 0) based on data obtained from the surface probe (open squares) and the myocardial probe (filled triangles). Panels (c) and (d) depicts paired comparisons of relative changes in pyruvate concentrations between the surface probe and the myocardial probe at corresponding time points (filled circles). Data are presented as mean \pm SEM (panels a and $\mathrm{b}$ ) and as mean $\pm 95 \%$ confidence intervals (panels $\mathrm{c}$ and $\mathrm{d}$ ). $\mathrm{E}=$ Equilibration time, $\mathrm{B}=$ Baseline, $\mathrm{I}=$ Ischaemia. Protocol $1, \mathrm{n}=10$ and protocol 2, $\mathrm{n}=8$.

et al., 2005). Equilibration time depends on biophysical properties of the specific substances and tissues that are assessed, as well as the physiological conditions guiding the fluid composition in the interstitial compartment. One clinical study has shown that equilibration time for glutamate was 30 min longer in injured patients than in healthy patients (Flodgren et al., 2005). Thus, there may be variability in equilibration time, both in health and injury, for different circulatory conditions, and for different organs/tissues and different substances. Our findings showed that for the substances analysed, the only substance with a shorter equilibrium for the surface probe compared with myocardial probe was glycerol. Glycerol is known as a marker for tissue damage (Paschen et al., 1986; Hillered et al., 1998). There is a possibility that tissue damage related to probe placement could contribute to higher glycerol values during the immediate equilibration period.

When there is placement of microdialysis probes within solid organs using needles, there is always risk of organ damage or bleeding. Local bleeding at the insertion site presumably increases time to adequate equilibration (Mantovani et al., 2002; Valen et al., 2004; Pöling et al., 2007a). Absence of damage when the probe is placed on the epicardial surface might result in reduced equilibration time. Microdialysis sampling with a conventional microdialysis probe on the surface probably will not be optimally effective for surface sampling, and increased contact surface as well as limitation of contact to other surfaces/compartments will allow more effective exchange. Stabilization of the contact and contact area of the membrane will also facilitate optimal membrane exchange.

There are several advantages to a surface position of a sampling probe. This application lowers the risk for bleeding complications compared to when probes are placed and removed from the myocardium, though clinical reports of microdialysis probes from the heart have not described complications (Bahlmann et al., 2004; Pöling et al., 2007a, 2008). The surface approach might allow probe placement and sampling even on thin-walled organs including for example the heart atrial muscle, where the intramyocardial approach is not practical. This type of sampling could, for example, facilitate better monitoring of the effects of cardioprotection during heart surgery.

To conclude, we present findings showing that surface microdialysis sampling identifies myocardial ischaemia and reperfusion. Surface heart microdialysis sampling is a novel application for microdialysis and ischaemia detection that shows promise and needs further investigation. A better probe construction more suitable for surface sampling is wanted.

\section{Conflicts of interest}

The authors declare no conflicts of interest 


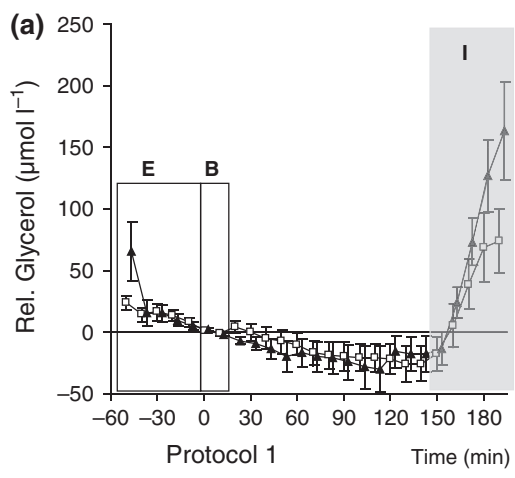

(c) Surface probe - Myocardial probe

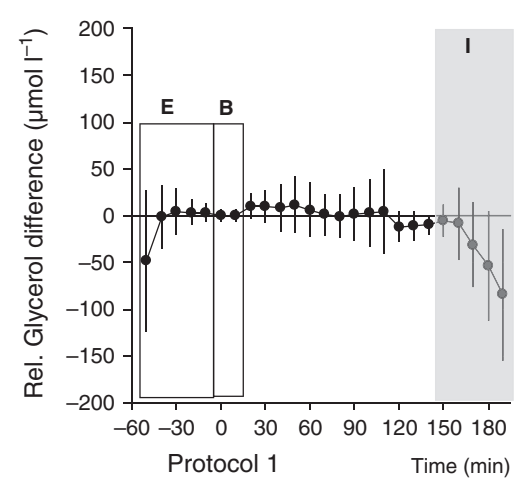

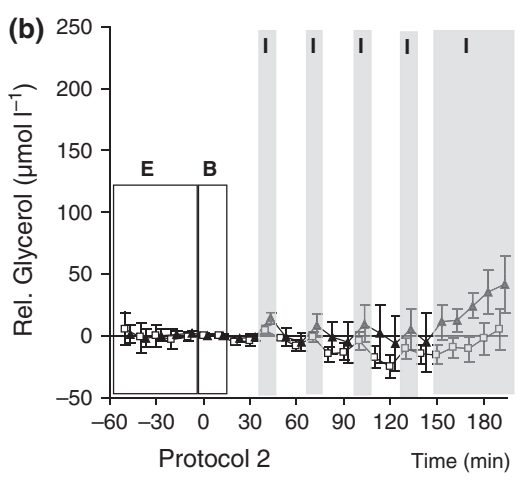

(d) Surface probe - Myocardial probe

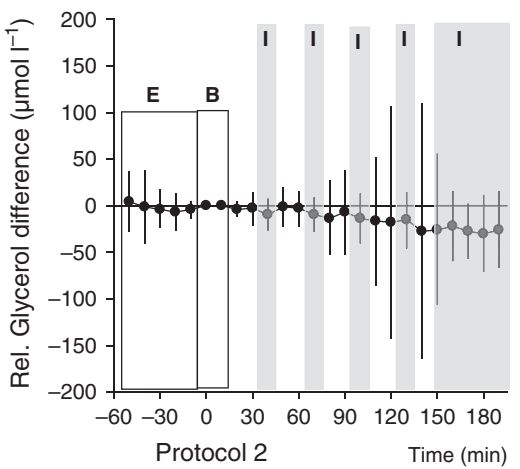

Figure 4 Glycerol. Panels (a) and (b) depicts relative changes in glycerol concentrations from baseline (time 0) based on data obtained from the surface probe (open squares) and the myocardial probe (filled triangles). Panels (c) and (d) depicts paired comparisons of relative changes in glycerol concentrations between the surface probe and the myocardial probe at corresponding time points (filled circles). Data are presented as mean \pm SEM (panels $\mathrm{a}$ and $\mathrm{b}$ ) and as mean $\pm 95 \%$ confidence intervals (panels $\mathrm{c}$ and $\mathrm{d}$ ). $\mathrm{E}=$ Equilibration time, $\mathrm{B}=$ Baseline, $\mathrm{I}=$ Ischaemia. Protocol $1, \mathrm{n}=10$ and protocol $2, \mathrm{n}=8$.

\section{References}

Abrahamsson P, Johansson G, Aberg AM, Haney M, Winso O. Optimised sample handling in association with use of the CMA 600 analyser. J Pharm Biomed Anal (2008); 48: 940-945.

Bahlmann L, Misfeld M, Klaus S, Leptien A, Heringlake M, Schmucker P, Sievers HH, Ungerstedt U, Kraatz EG. Myocardial redox state during coronary artery bypass grafting assessed with microdialysis. Intensive Care Med (2004); 30: 889-894.

Bolinder J, Hagström E, Ungerstedt U, Arner P. Microdialysis of subcutaneous adipose tissue in vivo for continuous glucose monitoring in man. Scand J Clin Lab Invest (1989); 49: 465-474.

Chaurasia CS. In vivo microdialysis sampling: theory and applications. Biomed Chromatogr (1999); 13: 317-332.

Flodgren GM, Crenshaw AG, Alfredson $\mathrm{H}$, Fahlstrom M, Hellstrom FB, Bronemo L, Djupsjobacka M. Glutamate and prostaglandin E2 in the trapezius muscle of female subjects with chronic muscle pain and controls determined by microdialysis. Eur J Pain (2005); 9: 511-515

Hillered L, Valtysson J, Enblad P, Persson L. Interstitial glycerol as a marker for membrane phospholipid degradation in the acutely injured human brain. J Neurol Neurosurg Psychiatry (1998); 64: 486-491.

Kavianipour M, Wikstrom G, Ronquist G, Waldenstrom A. Validity of the microdialysis technique for experimental in vivo studies of myocardial energy metabolism. Acta Physiol Scand (2003); 179: 61-65.

Kennergren C, Mantovani V, Strindberg L, Berglin E, Hamberger A, Lonnroth P. Myocardial interstitial glucose and lactate before, during, and after cardioplegic heart arrest. Am J Physiol Endocrinol Metab (2003); 284: E788E794.

Langemann H, Habicht J, Mendelowitsch A, Kanner A, Alessandri B, Landolt H, Gratzl O. Microdialytic monitoring during a cardiovascular operation. Acta Neurochir Suppl (1996); 67: 70-74
Mantovani V, Kennergren C, Berglin E, Moratti R, Lönnroth P, Hamberger A, Viganó $M$. Intramyocardial troponin-T monitoring with microdialysis in coronary artery bypass surgery. Scand Cardiovasc J (2002); 36: 308-312. Mantovani V, Kennergren C, Goiny M, Ungerstedt U, Lonnroth P, Sala A, Berglin E. Microdialysis for myocardial metabolic surveillance: developing a clinical technique. Clin Physiol Funct Imaging (2006); 26: 224-231.

Paschen W, van den Kerchhoff W, Hossmann K. Glycerol as an indicator of lipid degradation in bicuculline-induced seizures and experimental cerebral ischemia. Metab Brain Dis (1986); 1: 37-44.

Pöling J, Leptien A, Klaus S, Rees W, Kraatz E, Wiebe K, Bahlmann L. Analysis of the myocardial metabolism by microdialysis during open beating heart surgery. Scand Cardiovasc J (2007a); 41: 114-119.

Pöling J, Rees W, Klaus S, Bahlmann L, Hubner N, Mantovani V, Warnecke H. Myocardial metabolic monitoring with the microdialysis technique during and after open heart sur- 
gery. Acta Anaesthesiol Scand (2007b); 51: 341-346.

Pöling J, Rees W, Klaus S, Bahlmann L, Hubner N, Heringlake M, Mantovani V, Warnecke H. Functional recovery of chronic ischemic myocardium after surgical revascularization correlates with magnitude of oxidative metabolism. Cardiology (2008); 110: 174-181.
Stahl M, Bouw R, Jackson A, Pay V. Human microdialysis. Curr Pharm Biotechnol (2002); 3: 165-178.

Ungerstedt U. Microdialysis - principles and applications for studies in animals and man. J Intern Med (1991); 230: 365-373.

Valen G, Owall A, Takeshima S, Goiny M, Ungerstedt U, Vaage J. Metabolic changes induced by ischemia and cardioplegia: a study employing cardiac microdialysis in pigs. Eur J Cardiothorac Surg (2004); 25: 69-75. Waldenström A, Haney M, Biber B, Kavianipour M, Moritz T, Strandén P, Wikström G, Ronquist G. Ischaemic preconditioning relates to decreasing levels of extracellular adenosine that may be energy-metabolically useful in myocardium at risk; an experimental study in the pig. Acta Physiol (Oxf) (2010); 199: 1-9. 